\title{
An ensemble method to forecast 24-h ahead solar irradiance using wavelet decomposition and BiLSTM deep learning network
}

\author{
Pardeep Singla $^{1}$ (D) Manoj Duhan ${ }^{1} \cdot$ Sumit Saroha ${ }^{2}$
}

Received: 5 February 2021 / Accepted: 28 October 2021 / Published online: 17 November 2021

(c) The Author(s), under exclusive licence to Springer-Verlag GmbH Germany, part of Springer Nature 2021

\begin{abstract}
In recent years, the penetration of solar power at residential and utility levels has progressed exponentially. However, due to its stochastic nature, the prediction of solar global horizontal irradiance (GHI) with higher accuracy is a challenging task; but, vital for grid management: planning, scheduling \& balancing. Therefore, this paper proposes an ensemble model using the extended scope of wavelet transform (WT) and bidirectional long short term memory (BiLSTM) deep learning network to forecast 24-h ahead solar GHI. The WT decomposes the input time series data into different finite intrinsic model functions (IMF) to extract the statistical features of input time series. Further, the study reduces the number of IMF series by combining the wavelet decomposed components (D1-D6) series on the basis of comprehensive experimental analysis with an aim to improve the forecasting accuracy. Next, the trained standalone BiLSTM networks are allocated to each IMF subseries to execute the forecasting. Finally, the forecasted values of each sub-series from BiLSTM networks are reconstructed to deliver the final solar GHI forecast. The study performed monthly solar GHI forecasting for one year dataset using one month moving window mechanism for the location of Ahmedabad, Gujarat, India. For the performance comparison, the naïve predictor as a benchmark model, standalone long short term memory (LSTM), gated recurrent unit (GRU), BiLSTM and two other wavelet-based BiLSTM models are also simulated. From the results, it is observed that the proposed model outperforms other models in terms of root mean square error (RMSE) \& mean absolute percentage error (MAPE), coefficient of determination $\left(\mathrm{R}^{2}\right)$ and forecast skill (FS). The proposed model reduces the monthly average RMSE by range from 26.04$58.89 \%, 5.17-31.35 \%, 23.26-56.06 \%$ \& $21.08-57 \%$ in comparison with benchmark, standalone BiLSTM, GRU \& LSTM networks respectively. On the other hand, the monthly average MAPE is reduced by range from 9 to 51.18\%, 12.59-28.14\%, 30.43-59.19\% \& 26.54-58.92\% in comparison to benchmark, standalone BiLSTM, GRU \& LSTM respectively. Further, the proposed model obtained the value of $\mathrm{R}^{2}$ equal to 0.94 and forecast skill (\%) of $47 \%$ with reference to the benchmark model.
\end{abstract}

Keywords Solar irradiance forecasting · Deep learning $\cdot$ Long short term memory $\cdot$ Gated recurrent unit · Bidirectional long short term memory $\cdot$ Wavelet transform $\cdot$ Hyperparameters

Article Highlights

- A state of the art literature review of deep learning networks.

- Development of conventional LSTM, GRU and BiLSTM along

with naïve predictor.

- Implementation of wavelet transforms to the input data series.

- Selection of best wavelet IMF combinations to improve the accuracy of model.

- Ensemble of selected wavelet IMF combinations with BiLSTM deep learning network.

- Performance comparisons of proposed model with naïve, LSTM, BiLSTM, GRU, WT-BiLSTM (T) \& WT-BiLSTM (Mod).

Communicated by: H. Babaie

Pardeep Singla

pradeepsingla7@gmail.com

Extended author information available on the last page of the article

\section{Introduction}

Solar energy is one of the leading renewable energy resources (RER) to generate electricity with zero carbon emission (Wang et al. 2019) and its market is increasing rapidly from the last decade due to its sustainable \& supported characteristics (Chen et al. 2020). According to the international energy agency (IEA), by the end of 2030, the total capacity of the PV installation will hit the $1700 \mathrm{GW}$ level (Labouret and Villoz 2010). However, this power capacity was $8 \mathrm{GW}$ in 2007 and now reported $402 \mathrm{GW}$ in 2017, as per the global energy states report (REN 21) (Hales and Renewables 2018). Moreover, as per numerous investigations, the power grid will be $100 \%$ fully operational on 
the RER by the end of the year 2050 (Jacobson et al. 2017). But, due to the direct dependency of solar PV output over climatic \& geographical conditions, the stability of solar irradiance is always a subject of discussion in regard to grid management (Lan et al. 2019). Its inherent variable characteristics \& uncertainties must be accommodated by the resource planners to make a reliable grid- interconnected power system (Dong et al. 2020). Therefore, the precise and accurate forecasting of solar components is one of the prime requirements for two basic reasons. (a) to make the reliable grid-interconnected system which accommodates volatile, intermittent $\&$ random characteristics of solar generation, (b) to increase the utilization ratio of solar PV output along with maximum returns on the capital investment on solar park development (Zang et al. 2020a). However, reliable solar irradiance forecasting is not an easy task as it is very susceptible to seasonal and climatic effects. A minor fluctuation in the meteorological variables directly affects the stability \& reliability of the dependent systems (AlSkaif et al. 2020). In such situations, an appropriate forecasting model is highly requested. Several examinations have already been conducted on the development of methods to forecast the PV power or the solar irradiance components (Yu et al. 2019). In general, the forecasting methods can be divided into five different sections: based on process type, based on prediction method, based on spatial scale forecasting process, time horizon-based forecasting, prediction form-based forecasting, and method / approach-based forecasting (Lai et al. 2019). The different categories are shown in Fig. 1.

An approach-based forecasting is commonly used over various time horizons, systems, shapes and sizes (Lai et al. 2019). The persistence approach assumes the past similar day/h value of the target vector as the value of the presentday/h. If ' $t$ ' is the present time, then the value of target vector at ' $t$ ' time is considered as the value of ' $t+k$ ' where ' $k$ ' is the time horizon for which forecasting is being performed (Kumler et al. 2019). On the other hand, the physical approach generates the mathematical relationship between the target GHI to the meteorological \& geographical parameters. These models performed the forecasting of solar GHI by the use of meteorological and geographical variables instead of historical time series data. However, these models are not popular due to its high computational cost and worst precision (Alonso-Montesinos and Batlles 2015). European Centre for Medium-Range Weather Forecasts (ECMWF) \& Weather Research and Forecasting (WRF) models are the two popular models used for forecasting the atmospheric \& operational services (Richardson et al. 2020; Perez et al. 2012; Yang and Kleissl 2016).

Conversely, the statistical approach improves the accuracy and minimizes the error of the model by managing the correlation mapping between input and output parameters (Ruhang 2016). Grey theory (Li and Zhang 2019), Regression Analysis (Doorga et al. 2019), Fuzzy method (Reza Parsaei et al. 2020), time-series method (Bigdeli et al. 2017) and machine learning (ML) (Lai et al. 2020) are the different categories of statistical approach. The regression Auto Regressive Integrated Moving Average (ARIMA) techniques (Shadab et al. 2020), support vector regression (SVR) (Mohammadi and Aghashariatmadari 2020), Gaussian Progress Regression (GPR) (Sheng et al. 2018) and ML approach based models proved to be best in the recent years in terms of forecasting (Gao et al. 2020). ML approach such as artificial neural network (ANN) (Jahani and Mohammadi 2019), support vector machine (SVM) (Zeng and Qiao 2013), and Elman neural network (ENN) (Dumitru et al. 2016) were used historical data to learn the pattern which improves the accuracy of the model. Along with, a standalone model sometimes relies on the limited characteristics of the data and failed to learn hidden characteristics of data. This limited learning of the model leads to the poor performance of the model. To overcome this problem, hybrid models has been developed such as: PLA-k-means-HGWORF (Liu and Sun 2019), ANFIS-ANN (Kumar and Kalavathi 2018), ECMWF-ANN (Aguiar et al. 2016), SVM-RBF-WT (Shamshirband et al. 2016), WT-NNMFOA-GMDHMFOA (Heydari et al. 2019), WMIM-ELM (Cornejo-Bueno et al. 2019), LASSO-ANN (Huang et al. 2019) etc.

In addition, the deep learning approach emerges with great advantages with better accuracy for the forecasting of
Fig. 1 different categories of solar irradiance forecasting methods

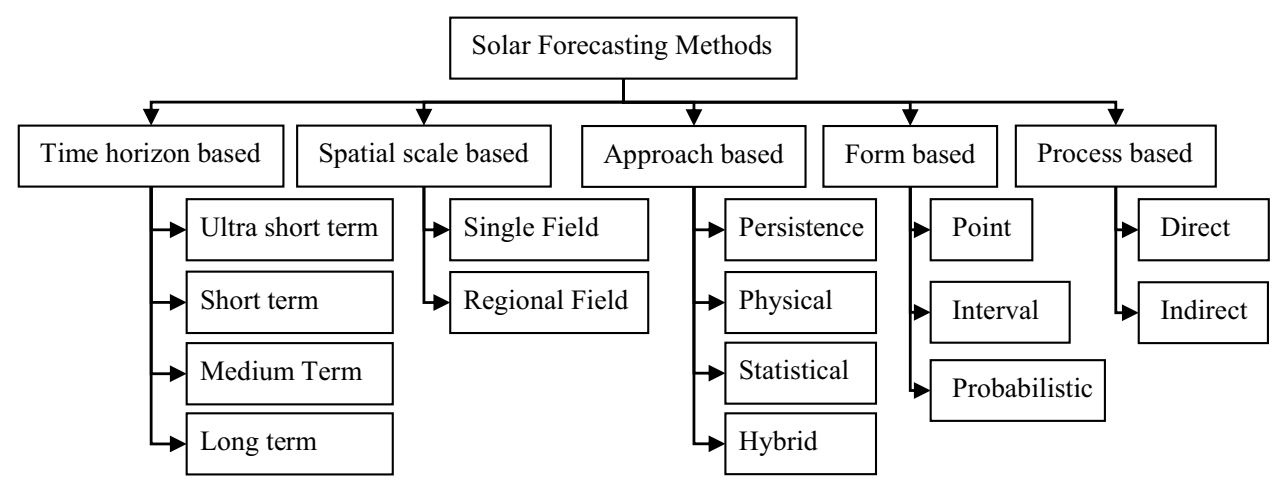


solar GHI. Due to its highest accuracy and great power of data processing abilities, this approach is dominating the ML approach (Bedi and Toshniwal 2019). Qing X. et al. forecasted the solar GHI on hourly basis using LSTM network in which weather data was used as an input feature of the LSTM network. The paper compared the performance of proposed network with the persistence, linear regression and back propagation neural network. The designed network outperformed the other model with RMSE of $122.71 \mathrm{~W} / \mathrm{m}^{2}$ and proved that the LSTM is better than ANN and other regression based models (Qing and Niu 2018). An ensemble method of deep learning and extreme gradient boosting was developed to avoid the problem of over fitting and compared with the persistence and SVR. The proposed model achieved the RMSE of $51.35 \mathrm{~W} / \mathrm{m}^{2}$ which was lesser in comparison to other models (Kumari and Toshniwal 2021). Likewise, the LSTM and convolution neural network (CNN) were combined by Zang $\mathrm{H}$. et al. to forecast the global solar irradiance. In this study, LSTM network used to acquire temporal features of the input dataset and $\mathrm{CNN}$ was used to fetch the spatiotemporal features (Zang et al. 2020b). A very short-term solar forecast using sky images was performed by Cong Feng et al. where, 20 layers, 13 convolution layers and five-max pooling layers with two fully connected layers based CNN model was developed to forecast the solar GHI. The model achieved $116.82 \mathrm{~W} / \mathrm{m}^{2}$ of RMSE, $-4.56 \mathrm{~W} / \mathrm{m}^{2}$ of MBE and $25.14 \%$ of forecast skill score for 1-h ahead prediction (Feng and Zhang 2020). Further, the combination of a residual network (ResNet) \& densed convolution network (DenseNet) was used by Hanxia et al. to forecast the 1-h ahead PV power. The variational mode decomposition was used as a preprocessing tool, to decompose the input dataset into trends, seasonal and random components (Zang et al. 2020a). The WT along with LSTM was utilized by Wang $\mathrm{F}$ et al. and the results proved that the models accuracy can be improved by WT (Wang et al. 2018). The WT and LSTM network was also used by M. Mishra et al. The study predicted the solar power for 1-h to 1-day ahead of time horizon in which WT was used to decompose the input raw data series into different frequency components and LSTM was used to forecast solar power (Mishra et al. 2020). In the same line, the GRU network was proposed by B. Gao et al. to forecast the day ahead solar irradiance. The model predicted the day ahead solar GHI with the combination of time series and meteorological data as an input. The GRU network provided the minimum RMSE of $122.45 \mathrm{~W} / \mathrm{m}^{2}$ with $42.01 \%$ of forecast skill (Gao et al. 2019).

Several other studies on deep learning are also available in the literature and most of them used LSTM, CNN for PV power forecasting \& wind forecasting (Ding et al. 2019; Kumar et al. 2020; Hu and Chen 2018). But, a few studies are available with the BiLSTM network to forecast the solar irradiance. However, BiLSTM network have already been used in electric load (Wu et al. 2020) $\&$ price forecasting (Cheng et al. 2019), tourism demand forecasting (Kulshrestha et al. 2020), user's next location forecasting (Bao et al. 2020), stock \& demand forecasting (Kim and Moon 2019), wind speed forecasting (Ding et al. 2019; Hu and Chen 2018) as well as covid-19 (Zeroual et al. 2020) predictions. With respect to BiLSTM, Li C. et al. predicted the solar irradiance using the standalone BiLSTM network. The BiLSTM network utilized the meteorological data to predict the hourly solar radiation. The results of the study clearly showed that the BiLSTM network is superior to the LSTM, SVR and LR models with RMSE of $98.44 \mathrm{~W} / \mathrm{m}^{2}$, MAE of $71.49 \mathrm{~W} / \mathrm{m}^{2}(\mathrm{Li}$ et al. 2021) . Rai A. et al. predicted the solar radiations for the time horizon of two months using the CNN and BiLSTM. The CNN was used to extract the features of the input data time series; whereas, BiLSTM exploited the dependencies of the time series. This study proved that the CNN-BiLSTM model performed better than LSTM, GRU, CNN-LSTM and GRU-LSTM (Rai et al. 2021). Therefore, inspired from the above work, this paper proposes an ensemble model to forecast 24-h ahead solar GHI using wavelet transform (WT) and BiLSTM with an objective to improve forecasting accuracy. For the justification of superiority of proposed model, the performance is compared with the reproduced naïve predictor and other proven deep learning networks such as: unidirectional LSTM, unidirectional GRU, BiLSTM, traditional wavelet-based BiLSTM (WT-BiLSTM(T)) and modified wavelet-based BiLSTM (WT-BiLSTM(Mod)).

In brief, the major contributions are as follows:

- A brief literature of solar forecasting based on deep learning network by considering almost all important factors related to forecasting.

- Reproduction of naïve predictor (benchmark), three different standalone deep learning models: unidirectional LSTM, unidirectional GRU and BiLSTM network.

- With the aim to fill the space of WT-BiLSTM process, the various scenarios of WT based BiLSTM models are analyzed. For this, the traditional WT is combined with BiLSTM (WT-BiLSTM (T)) network to forecast the solar GHI. In the other scenario, the scope of WT is extended by combining the different wavelet decomposed subseries (IMF) and fed to BiLSTM network (WT-BiLSTM (mod)) to forecast solar GHI.

- Implementation of proposed model based on forecasting of wavelet decomposed component using BiLSTM (WT-BiLSTM(CF)). The proposed model is developed to improve the forecasting using the best use of WT with BiLSTM. The model allocates an individual BiLSTM network to the best combination of wavelet decomposed subseries. The forecasted value obtained from BiLSTM 
for each subseries are reconstructed to generate the final forecast.

- Performance analysis of proposed model in comparison with other reproduced models along with benchmark model. The proposed model observed as a best performer among all models with lesser annual RMSE of $45.61 \mathrm{~W} /$ $\mathrm{m}^{2}$, MAPE of $6.48 \%$ and $\mathrm{R}^{2}$ of 0.94 . The forecast skill of the proposed model against naïve predictor is also observed and found $47 \%$.

Therefore, the paper is structured in such a way that the theoretical context of the wavelet transformation, LSTM and BiLSTM deep learning network is defined in Section 2. Section-3 deals with the framework of the suggested model while Section-4 addresses various experimental scenarios. All experiments are analyzed as a discussion in section-5. Finally, the conclusion is drawn in section-6.

\section{Theoretical background}

This section provides a brief overview of the pre-processing i.e. WT and deep learning networks related to the proposed forecasting model.

\section{Wavelet transform (WT)}

It is a tool to process the highly random \& time-varying data signals by which time domain signal can be directly transformed into the time-scaled frequency domain (Zhang et al. 2020). In other words, WT decomposes nonlinear and the non-stationary data in to set of different frequency profiles. These frequency profiles extract the appropriate statistical characteristics of the input data series. The high frequency components provide the short term variations in the data and can be used to improve the forecasting accuracy for short term prediction. The WT can be categorized into two types according to the input signal: Continuous wavelet transform (CWT) and Discrete wavelet transform (DWT) (El-Hendawi and Wang 2020). This CWT can be expressed mathematically as:

$W_{C W T}(a, b)=a^{-1 / 2} \int_{-\infty}^{\infty} G H I(t) \psi\left(\frac{t-b}{a}\right) d t$

where, ' $W_{C W T}$ ' is CWT, ' $G H I(t)$ ' is global horizontal irradiance time series, ' $\psi(t)$ ' is mother wavelet which is scaled \& time shifted by ' $a$ ' \& ' $b$ ' respectively.

Whereas, the DWT for a time series GHI (t); $t=1,23$, can be expressed as a mother wavelet ' $\psi_{m, k}$ ' \& father wavelet ' $\varphi_{m, k}$ ' and expressed mathematically as (El-Hendawi and Wang 2020): $\psi_{m, k}(t)=\left(2^{m}\right)^{1 / 2} \psi\left(2^{m}-t\right)$

$\varphi_{M, k}(t)=\left(2^{M}\right)^{1 / 2} \varphi\left(2^{M}-t\right)$

In DWT, the convolution of father wavelet ' $\varphi$ ' with original series ' $G H I(t)$ ' gives the approximation components ' $A_{m, k}$ '. Whereas, the convolution of mother wavelet ' $\psi$ ' with original series ' $G H I(t)$ ' gives the detail components ' $D_{m, k}$ '.

So, $A_{M, k}=\int_{-\infty}^{\infty} G H I(t) \varphi_{M, k} d t$

$D_{m, k}=\int_{-\infty}^{\infty} G H I(t) \psi_{m, k} d t$

However, $G H I(t)=\sum_{k e Z} A_{M, k} \varphi_{M, k}(t)+\sum_{m=1}^{M} D_{m, k} \psi_{m, k}(t)$

Or in simple terms, for a finite length of series with finite decomposition is (Saroha and Aggarwal 2018):

$G H I(t)=D 1+D 2+D 3+\cdots+D n+A 1+A 2+A 3+\cdots+A n$

Where "D1, D2, D3 ,..., Dn" are the detailed components of input time series and "A1, A2, A3,..,An" are the approximation components of input time series.

\section{Long short-term memory network (LSTM)}

For processing the sequence data, J. J. Hopfield proposed a structure in 1982, called Recurrent Neural Network (RNN). Unlike conventional ANN, the output of RNN is connected back to the input through feedback which acts as dynamic memory (Zang et al. 2020b). This network performed best for the short term forecasting but becomes unstable for long term forecasting. This instability is due to exploding of gradient i.e. sudden large variations in training weights (Hochreiter and Schmidhuber 1997). The solution of gradient exploding was provided by LSTM network by allowing memory cells in the hidden layer(s). These memory cells are used to appropriately store the information or discard the relevant information of the data. The basic architecture of the LSTM is shown in Fig. 2.

Each cell of the LSTM consists of forget gate $\left(f_{t}\right)$, input gate $\left(i_{t}\right)$ and output gate $\left(O_{t}\right)$ to accept or discard of any information (Kulshrestha et al. 2020). For a forward movement function, the previous cell state ' $C_{t-1}$ ' has been discarded by the network. At present time ' $t$ ', the LSTM 


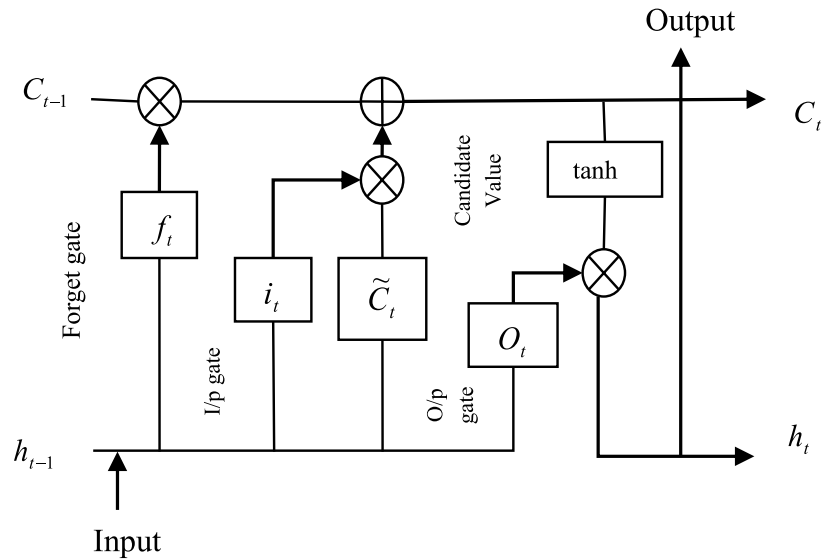

Fig. 2 LSTM architecture

network have three inputs ' $G H I(t)$ ', previous memory cell output ' $h_{t-1}$ ' and bias ' $b_{f}$ ' of forget gate (Fischer and Krauss 2018). Therefore, the activation values can be expressed as:

$f_{t}=\sigma\left(W_{f} \cdot\left[h_{t-1}, G H I_{i}(t)\right]+b_{f}\right)$

The following equations are used by the network to decide whether the information about data has to be discarded or retained.

$i_{t}=\sigma\left(W_{i \cdot} \cdot\left[h_{t-1}, G H I_{i}(t)\right]+b_{i}\right)$

$\tilde{C}_{t}=\tanh \left(W_{c} \cdot\left[h_{t-1}, G H I_{i}(t)\right]+b_{c}\right)$

$C_{t}=f_{t} * C_{t-1}+i_{i} * \tilde{\tilde{C}}_{t}$

Now, the final output of the memory cell becomes:

$O_{t}=\sigma\left(W_{o} \cdot\left[h_{t-1}, G H I_{i}(t)\right]+b_{o}\right)$

$h_{t}=O_{t} * \tanh \left(C_{t}\right)$

where " $b_{i}$ ", " $b_{f}$ ", " $b_{c}$ " $\&$ " $b_{o}$ " are bias vectors of LSTM network; " $W_{i}$ ", " $W_{f}$ ", " $W_{c}$ " \& " $W_{o}$ " are weight vectors of LSTM network and $\sigma$ is sigmoid function ranges from ' $O$ ' to ' 1 '.

\section{Bidirectional long short-term memories (BiLSTM)}

The BiLSTM network is composed of forward and backward LSTM in which data can be processed in forward as well as in backward direction. The backward direction processing captures the hidden characteristics and pattern of the data which was generally ignored by LSTM (Yildirim 2018). The

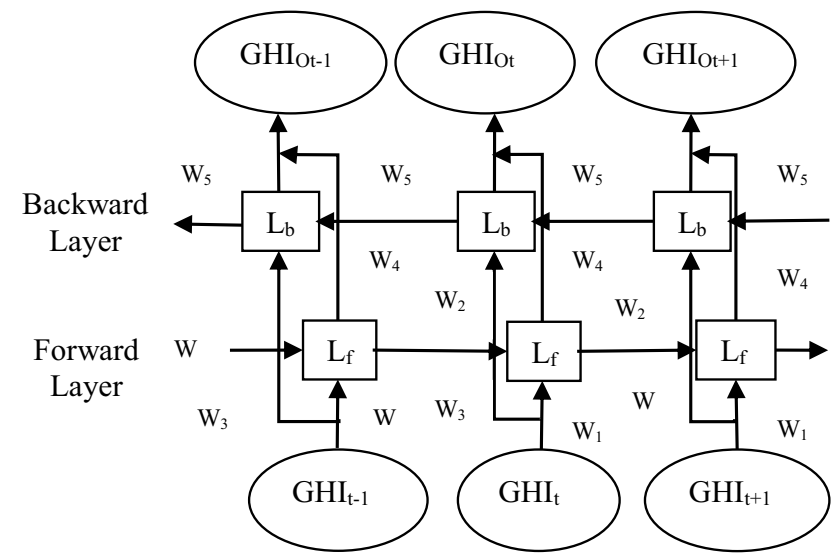

Fig. 3 BiLSTM architecture

basic architecture of BiLSTM network is shown in Fig. 3 (Li et al. 2021).

The forward hidden layer ' $L$ ', the backward hidden layer ' $L_{b}$ ' and output sequence ' $G H I_{o}(t)$ ' used to update the network. The network updates iteratively in backward i.e. from ' $T$ ' to ' $I$ ' and forward direction i.e. ' $I$ ' to ' $T$ '. The updated parameters of the network can be expressed mathematically as:

$L_{f}=\sigma\left(W_{1} G H I_{i}(t)+W_{2} L_{f-1}+b_{L_{f}}\right)$

$L_{b}=\sigma\left(W_{3} G H I_{i}(t)+W_{5} L_{b-1}+b_{L_{b}}\right)$

$G H I_{o}=W_{4} L_{f}+W_{6} L+b_{G H I_{o}}$

Where, " $L_{f}$ ", " $L_{b}$ " \& " $G H I_{o}(t)$ " are forward pass, backward pass and final output layers respectively. ' $W$ ' is the weights coefficient and ' $b_{L_{f}}$ ', ' $b_{L_{f}}$ '\& ' $b_{G H I_{o}}$ ' are the biases (Cheng et al. 2019).

\section{Structure of proposed WT based BiLSTM model}

The basic idea of this work is to improve the forecasting accuracy of Solar GHI using WT based BiLSTM network in which different scenarios of WT pre-processing are being applied. The Fig. 4 shows the pictorial representation of proposed model and description of each step is given below:

A) Quality control of data: Initially, the collected data from any site is available in the raw form and has a great influence on the model's performance. There might be possibilities of presence of negative or incomplete data records due to weak pyranometer response. So, these 


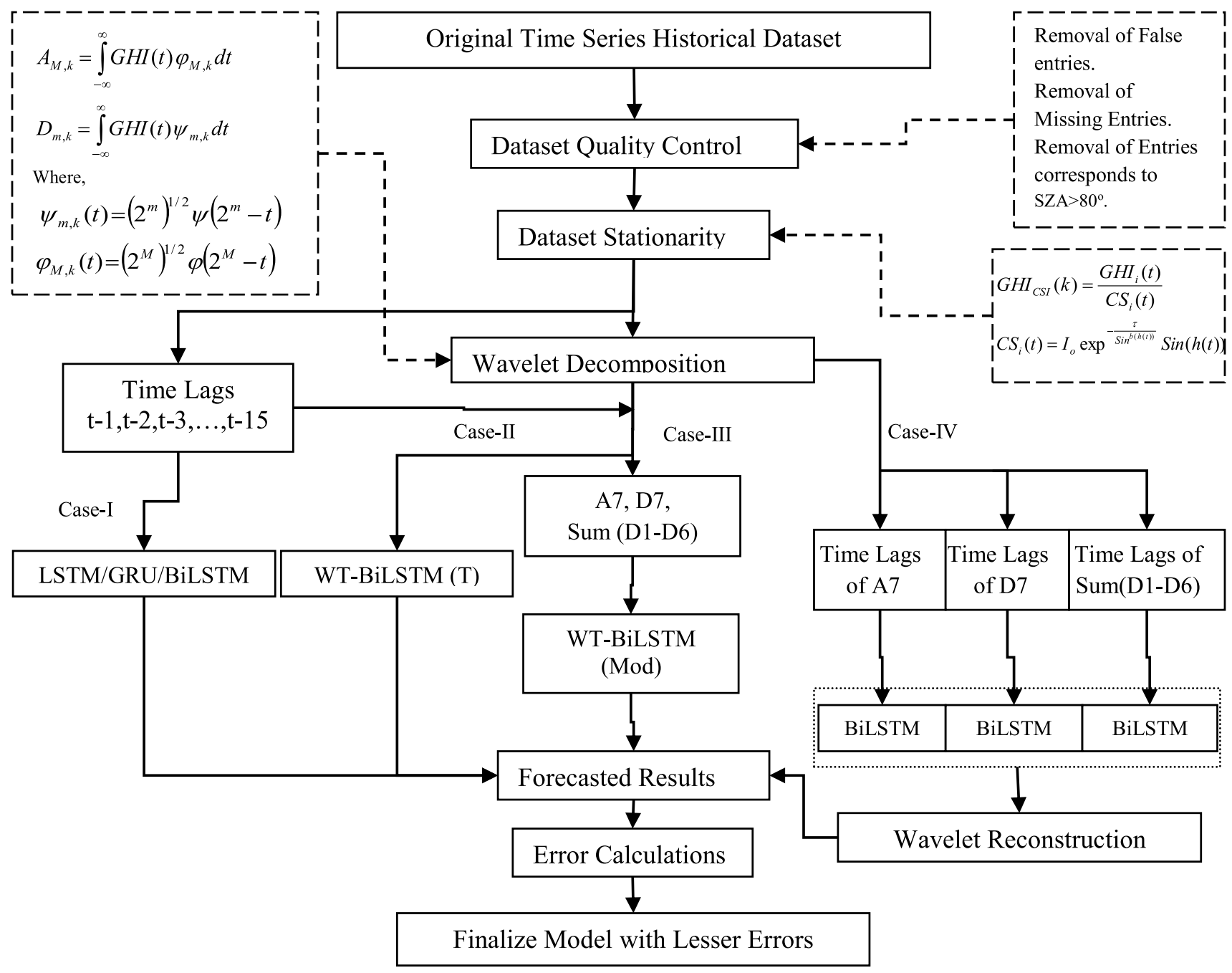

Fig. 4 Structure of proposed forecasting model

entries must be removed or corrected before the application to the forecasting model (Yousif et al. 2013). In addition to this, the night hours data are also removed from the dataset due to absence of solar irradiations in night time. The data just before the sunset and just after the sunrise is also a culprit factor in the model's performance due to cosine error of instruments and must be removed. In a single statement, this process can also be understood as the removal of data having solar zenith angle greater than $80^{\circ}$ (Lauret et al. 2015).

B) Data Stationarity: The majority of the solar data collected from the site is often random and nonlinear in nature which consists of periodicity and seasonality (Singh et al. 2018). Therefore, to enhance the model's efficiency, the data has to be rendered stationary before providing to the forecasting model. This paper considers the clear-sky index (CSI) calculation to render the data stationary or to turn in the " $0-1$ " range. The CSI can be calculated as (Benali et al. 2019):

$G H I_{C S I}(t k)=\frac{G H I_{i}(t)}{C S_{i}(t)}$

where, $C S_{i}(t)=I_{o} \exp ^{-\frac{\tau}{\operatorname{Sin}^{b(t(t))}}} \operatorname{Sin}(h(t))$

where $h(t)$ is height of solar (degree).

$\mathrm{I}_{\mathrm{o}}=$ Extra-terrestrial irradiation.

$\mathrm{b}=$ parameter of fitting.

Most research, however, have already shown that the clear sky GHI is nearly identical to the CS and can be replaced with each other, so

$k=\frac{G H I_{i}(t)}{G H I_{c s}}$

where, $\mathrm{GHI}_{\mathrm{cs}}=$ clear-sky GHI. 
III) Data decomposition: Using data quality control, once the time series has been cleaned \& converted into CSI. The WT is applied on the input data series to decompose into approximation and detail components. This work observed the Daubechies (db7) wavelet provides the required smoothness of considered data. However, the wavelet decomposition up to level 1-10 was observed experimentally where level-7 with $\mathrm{db} 7$ found best for the dataset used in the study. The pictorial representation of level-7 decomposition is shown in Fig. 5; whereas, Fig. 6 represents the wavelet decomposition results for the original solar GHI time series.

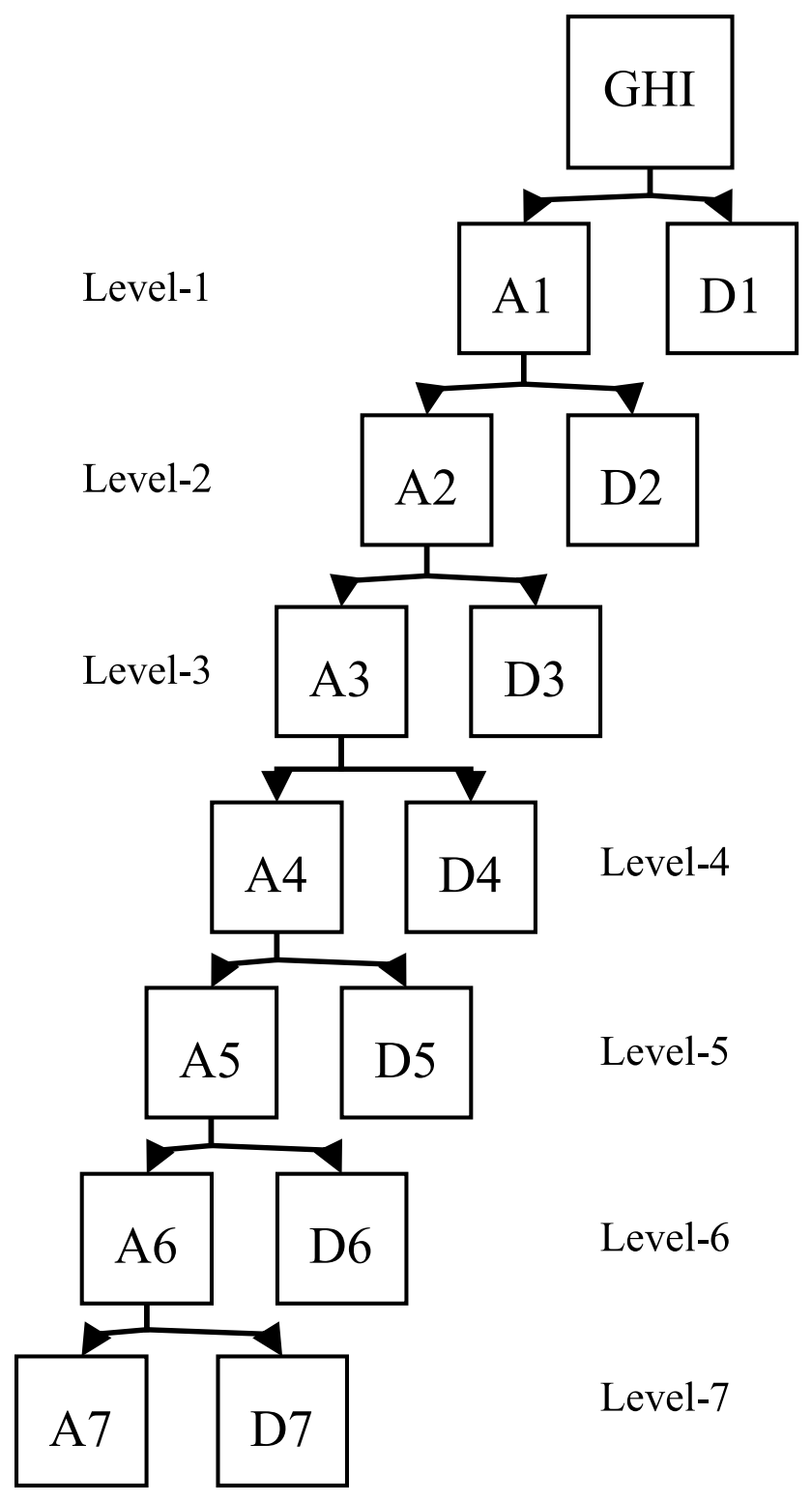

Fig. 5 Level-7 decomposition using discrete wavelet transform
Here, $G H I(t)=D 1+D 2+D 3+D 4+D 5+D 6+D 7+A 7$

IV) Selection of hyperparameters: One of the significant tasks in case of a deep learning algorithm is the tuning of hyperparameters. However, no such thumb-rule is present in the literature to set these hyperparameters at optimal range. But, the study obtained the optimal hyperparameters using the grid search algorithm by varying each parameter's value with in a specific bound. The specific process of searching the best hyperparameter is shown in Fig. 7 using a flow graph whereas, Table 1 provides the best values along with their corresponding bounds. This study kept these fine-tuned hyperparameters constant throughout the work to maintain the fairness in the comparison of different models. The specific rules to select best hyperparameters are mentioned below:

1) Set the initial hyperparameter at a default value.

2) Set the optimum learning rate.

3) Choose the perfect epoch with batch size.

4) Choose the relevant optimizer.

5) Select the appropriate activation function.

6) Choose suitable numbers of the hidden layer.

E) Forecasting Process: Input data is decomposed at level 7 using db7 wavelet in which eight components: A7 and D7-D1 are obtained. So, as input features, the eight components D1 to D7 and A7 with sufficient time lags are configured. This study performed a comprehensive experiment to obtain best solar GHI forecast for different combinations of decomposed components as input to the predictor for different level's decomposition (refer Table 5). For the same, the input dataset have been divided into two phases: the training phase and forecasting phase. With the aid of one year training data, forecasting is performed on monthly basis up-to next 12 months with moving window mechanism. The proposed model forecasts 24-h ahead of hourly data for each month. The output sequence is reconstructed to have the actual CSI at this stage. Although, using the following equation, this sequence is further transformed into real solar GHI from CSI.

$G H I(t)=C S(t) \times C S I$

Where, "CS(t)" \& "CSI" are Clear sky GHI \& clear sky index respectively. 
Fig. 6 Level-7 wavelet decomposition results
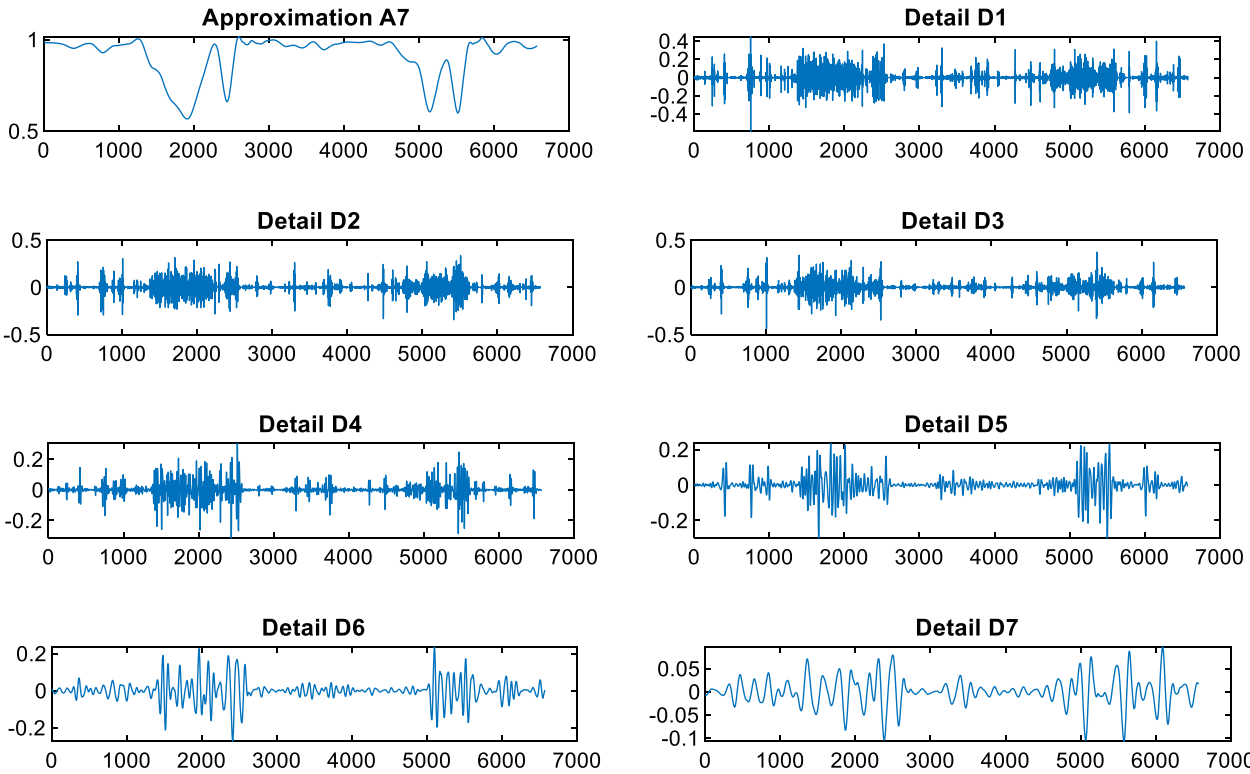

F) Performance Calculations:

Mean absolute percentage error (Sobri et al. 2018):

$M A P E=\frac{1}{n} \sum_{i=1}^{n}\left|\frac{G H I_{i}-G H \tilde{I}_{i}}{G H I_{i}}\right|$

where "GHI" is forecasted value of GHI at ' ${ }^{t h}$ ' level whereas, GHI is observed value of GHI.

Root Mean Square Error (Sobri et al. 2018):

$R M S E=\sqrt{\frac{1}{n} \sum_{i=1}^{n}\left(G H I_{i}-G H I_{i}\right)^{2}}$

Coefficient of determination $\left(\mathrm{R}^{2}\right)$ :

It used to represent the goodness of fit of a model and it is square of correlation coefficient ranges from 0 to 1 .

$R^{2}=1-\frac{\sum_{i=1}^{n}\left(G H I_{i}-G H \tilde{I}_{i}\right)^{2}}{\sum_{i=1}^{n}\left(G H I_{i}-\operatorname{mean}\left(G H I_{i}\right)\right)^{2}}$

Forecast Score (FS):

The percentage improvement in the forecasting by a developed model w.r.t. reference model is demonstrated by FS.

$F S=1-\frac{\text { Error Indicator }_{\text {proposedmethod }}}{\text { Error Indicator } \text { ref.method }}$

\section{Simulation results}

This work originated with the wavelet \& BiLSTM network in order to enhance the accuracy of forecasts. The performance of proposed model has been compared with some other standard models such as: naïve predictor, standalone deep learning networks (LSTM, GRU \& BiLSTM) and other WT based models. The simulation is performed in MATLAB 2020 environment where different scenarios of the models have been evaluated. To suggest an accurate model, the various simulations are performed as follows:

1) Naïve predictor, unidirectional LSTM, GRU and BiLSTM network.

2) Traditional wavelet decomposition technique with BiLSTM (WT-BiLSTM (T)).

3) Modified wavelet decomposition technique with BiLSTM (WT-BiLSTM (Mod)).

4) Proposed wavelet decomposition forecasted technique, which summed all forecasted decomposed components (WT-BiLSTM (CF)), where CF means for component forecasted.

5) Evaluation of forecasting performance of proposed model using RMSE, MAPE, $\mathrm{R}^{2} \& \mathrm{FS}$ and compared with naïve, LSTM, GRU, BiLSTM, WT-BiLSTM(T) and WT-BiLSTM(mod) models.

\section{Data description}

Because of the large investments in the renewable sector and exponentially increasing scope in India, this work considers the Indian location data set for the forecasting. This work utilizes the hourly data gathered from NSRDB, for 


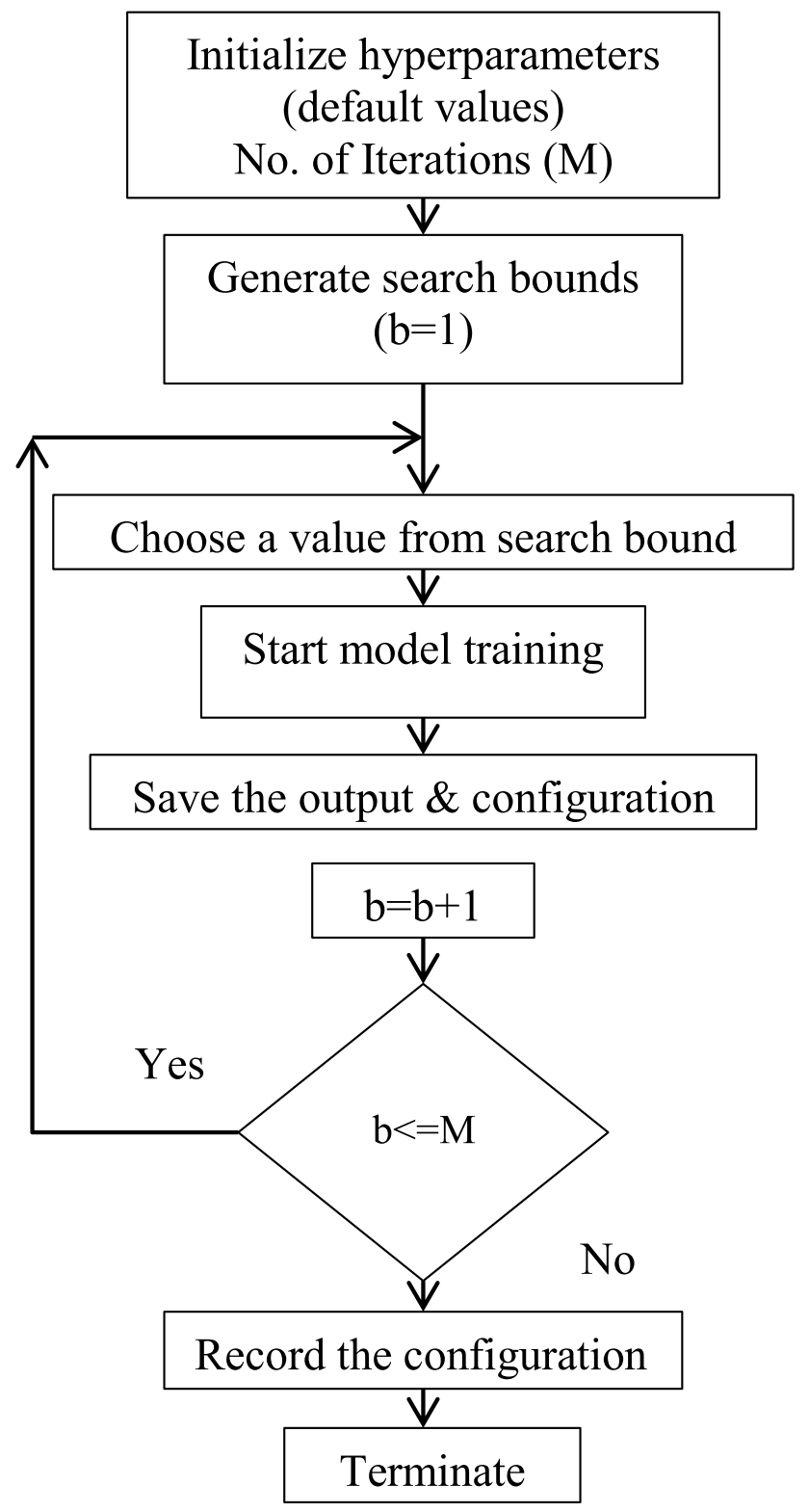

Fig. 7 Flow graph for selecting hyperparameters

Ahmadabad city situated in northern Gujarat, India with latitude / longitude of $23^{\circ} .03 \mathrm{~N} / 72^{\circ} .58 \mathrm{E}$ with an average elevation of $53 \mathrm{~m}$. According to the Koppen climate classification, the studied location has mixed kind of climate with subtropical hot steppe ('bsh') and humid subtropical ('cwa').
The collected dataset are the hourly recordings of different meteorological parameters along with historical solar GHI. However, a dataset of year 2013 \& 2014 have been used to train, validate and test the proposed model. The entire dataset was split into different datasets: training dataset $(80 \%)$, validation dataset (10\%) and test dataset (10\%). The study used the dataset of year 2013 for the training purpose while the next year data have been carried out to evaluate the model using monthly forecasting.

\section{Experimental setup}

All the developed models including proposed model are evaluated in terms of error (RMSE, MAPE), coefficient of determination $\left(\mathrm{R}^{2}\right)$ and forecasting skill (FS). To implement the improved \& accurate forecasting model, different scenarios of experiments have been conducted. The RMSE, MAPE \& $R^{2}$ for all scenarios are recorded in Tables 2, 3, and 4 and are discussed below:

\# Scenario 1: Forecast using naïve, LSTM, GRU \& BiLSTM

This experiment is all about the development of benchmark \& standalone deep learning models such as: Naïve, standalone LSTM, GRU and BiLSTM. The experiment is conducted for the forecasting of different month-wise 24-h ahead solar GHI where input initialization and finetuning of the hyperparameters of deep learning networks is one of the important tasks to achieve the better accuracy. The 15 time lags of input data series are provided to these deep learning based standalone models as an input features; whereas, the solar GHI is used as an output feature. Finally, the value of MAPE obtained by naïve, standalone LSTM, GRU and BiLSTM ranges from $2.41 \%-30.03 \%$, $3.31-32.97 \%, 3.66-33.18 \%$, and $2.51-18.96 \%$ respectively; RMSE ranges from $32.02-185.44 \mathrm{~W} / \mathrm{m}^{2}, 40.33-161.84 \mathrm{~W} /$ $\mathrm{m}^{2}, 32.73-164.01 \mathrm{~W} / \mathrm{m}^{2}$ and $30.94-113.06 \mathrm{~W} / \mathrm{m}^{2}$ respectively. Moreover, the correlation coefficient $\mathrm{R}^{2}$ ranges from $0.33-0.95,0.40-0.95$ and $0.41-0.97$ for naïve, standalone LSTM, GRU and BiLSTM respectively. Figure 8 shows the annual average RMSE \& MAPE comparison of this scenario. From these observations, It is clear that the BiLSTM

Table 1 Hyperparameters values used in the study

\begin{tabular}{|c|c|c|c|c|c|}
\hline Hyperparameter & Searched range & Value & Hyperparameter & Searched range & Value \\
\hline Hidden Units & $50-120$ & 100 & Epochs & $100-600$ & 500 \\
\hline Initial Learning Rate & $0.0001-0.1$ & 0.0007 & Optimizer & adam, sgdm, RMSprop & Adam \\
\hline Learning Rate Drop Period & $50-150$ & 125 & Learning Rate Drop Factor & Default & 0.2 \\
\hline
\end{tabular}


Table 2 RMSE $\left(\mathrm{W} / \mathrm{m}^{2}\right)$ comparison of developed models

\begin{tabular}{|c|c|c|c|c|c|c|c|c|c|c|c|c|c|}
\hline Models & Jan & Feb & Mar & Apr & May & Jun & July & Aug & Sep & Oct & Nov & Dec & Annual \\
\hline Naïve & 56.85 & 78.70 & 45.95 & 32.02 & 54.14 & 87.23 & 168.46 & 185.44 & 158.09 & 69.74 & 47.33 & 52.03 & 86.33 \\
\hline LSTM & 40.33 & 57.60 & 45.16 & 43.61 & 58.63 & 82.95 & 161.72 & 161.84 & 141.35 & 68.40 & 59.58 & 49.40 & 80.88 \\
\hline GRU & 42.81 & 56.36 & 39.70 & 32.73 & 48.87 & 71.55 & 164.01 & 163.64 & 149.35 & 60.60 & 54.93 & 39.79 & 77.03 \\
\hline BiLSTM & 33.57 & 44.94 & 37.46 & 31.92 & 49.25 & 65.97 & 88.90 & 113.06 & 84.51 & 56.94 & 34.94 & 30.94 & 56.03 \\
\hline WT-BiLSTM (T) & 32.52 & 40.69 & 32.67 & 29.86 & 44.72 & 61.21 & 84.10 & 107.17 & 79.80 & 50.64 & 32.40 & 25.53 & 51.77 \\
\hline WT-BiLSTM (Mod) & 31.91 & 40.41 & 31.46 & 29.96 & 43.08 & 58.60 & 81.19 & 106.65 & 74.16 & 49.16 & 34.02 & 23.78 & 50.36 \\
\hline Proposed Model & 31.83 & 35.69 & 26.85 & 23.26 & 36.46 & 52.84 & 85.61 & 96.38 & 65.78 & 46.50 & 24.96 & 21.24 & 45.61 \\
\hline
\end{tabular}

Table 3 MAPE (\%) comparison of developed models

\begin{tabular}{lccccccccccccc}
\hline Models & Jan & Feb & Mar & Apr & May & Jun & July & Aug & Sep & Oct & Nov & Dec & Annual \\
\hline Naïve & 6.43 & 7.68 & 3.02 & 2.41 & 4.92 & 10.97 & 28.55 & 30.03 & 28.07 & 7.35 & 6.72 & 4.83 & 11.74 \\
LSTM & 5.98 & 7.13 & 3.74 & 3.31 & 5.93 & 10.19 & 31.39 & 29.35 & 32.97 & 7.96 & 8.80 & 5.70 & 12.70 \\
GRU & 6.59 & 6.97 & 4.43 & 3.66 & 6.43 & 9.66 & 31.92 & 29.80 & 33.18 & 8.02 & 8.10 & 5.38 & 12.84 \\
BiLSTM & 3.77 & 4.99 & 2.93 & 2.51 & 5.57 & 8.72 & 16.23 & 18.96 & 16.29 & 6.38 & 5.88 & 4.07 & 8.02 \\
WT-BiLSTM (T) & 3.66 & 4.51 & 2.69 & 2.39 & 5.17 & 8.11 & 15.21 & 17.11 & 16.17 & 5.81 & 5.52 & 3.56 & 7.49 \\
WT-BiLSTM (Mod) & 3.43 & 4.40 & 2.62 & 2.39 & 5.03 & 7.76 & 14.23 & 17.46 & 15.28 & 5.84 & 5.59 & 3.34 & 7.28 \\
Proposed Model & 3.30 & 3.86 & 2.26 & 2.02 & 4.36 & 6.72 & 14.18 & 14.66 & 13.54 & 5.54 & 4.22 & 3.11 & 6.48 \\
\hline
\end{tabular}

Table $4 \mathrm{R}^{2}$ comparison of developed models

\begin{tabular}{lccccccccccccc}
\hline Models & Jan & Feb & Mar & Apr & May & Jun & July & Aug & Sep & Oct & Nov & Dec & Average \\
\hline Naïve & 0.91 & 0.86 & 0.95 & 0.93 & 0.92 & 0.78 & 0.46 & 0.33 & 0.56 & 0.88 & 0.93 & 0.92 & 0.78 \\
LSTM & 0.95 & 0.92 & 0.95 & 0.95 & 0.90 & 0.80 & 0.43 & 0.40 & 0.58 & 0.88 & 0.90 & 0.92 & 0.80 \\
GRU & 0.96 & 0.93 & 0.97 & 0.97 & 0.94 & 0.84 & 0.43 & 0.41 & 0.57 & 0.90 & 0.94 & 0.95 & 0.82 \\
BiLSTM & 0.97 & 0.95 & 0.97 & 0.96 & 0.94 & 0.87 & 0.82 & 0.69 & 0.86 & 0.92 & 0.96 & 0.97 & 0.91 \\
WT-BiLSTM (T) & 0.97 & 0.96 & 0.97 & 0.97 & 0.94 & 0.88 & 0.84 & 0.72 & 0.87 & 0.93 & 0.96 & 0.98 & 0.91 \\
WT-BiLSTM (Mod) & 0.97 & 0.96 & 0.98 & 0.97 & 0.95 & 0.89 & 0.85 & 0.73 & 0.89 & 0.94 & 0.96 & 0.98 & 0.92 \\
Proposed Model & 0.97 & 0.97 & 0.98 & 0.98 & 0.96 & 0.92 & 0.86 & 0.78 & 0.92 & 0.95 & 0.98 & 0.98 & 0.94 \\
\hline
\end{tabular}

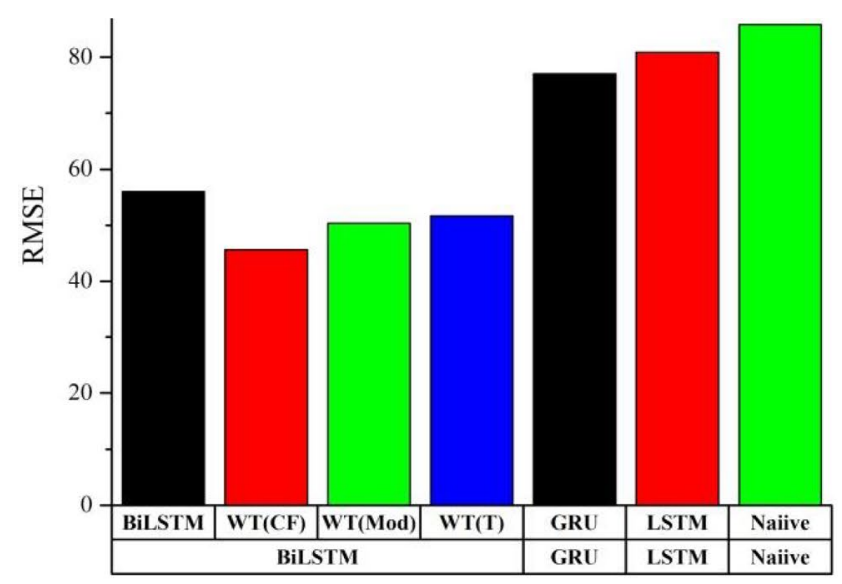

(a)

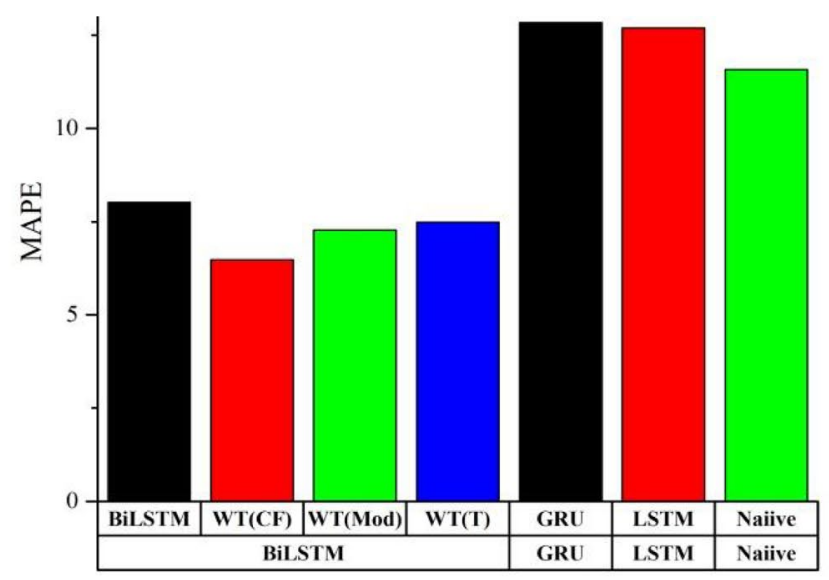

(b)

Fig. 8 Annual average RMSE (W/m²) \& MAPE (\%) of different models 
network outperforms over LSTM \& GRU as is also claimed in various previous studies (Li et al. 2021; Rai et al. 2021).

\section{\# Scenario 2: Forecast using traditional WT and BiLSTM} (WT-BiLSTM (T))

The traditional WT is used in this experiment to decompose the input data time series. This experiment utilizes all the decomposed components of WT along with the fifteen time lags of input data as input features of the BiLSTM model. The results in the Tables 2, 3 and 4 shows that the WT-BiLSTM(T) achieved lesser RMSE, MAPE and improved $\mathrm{R}^{2}$ in comparison to standalone LSTM, GRU and BiLSTM networks. This model achieved MAPE ranges from 2.39-17.77\%, RMSE ranges from $25.53-107.17 \mathrm{~W} / \mathrm{m}^{2}$ and $\mathrm{R}^{2}$ ranges from $0.72-0.98$. Moreover, the average annual RMSE, MAPE and $\mathrm{R}^{2}$ obtained from the same is 51.77 (W/ $\mathrm{m}^{2}$ ), $7.49 \%$ and 0.91 respectively.

\# Scenario 3: Forecast using Modified wavelet decomposition and BiLSTM (WT-BiLSTM (Mod))

Unlike, the traditional wavelet where all decomposed components were used as input features to the BiLSTM network. This experiment employs the different combinations of decomposed components as an input feature with the time lag input data. A thorough analysis has been done to observe the effect of these combinations of decomposed components as an input feature to the performance of model. Table 5 shows the observations recorded for different combinations of decomposed components as input features. Here, the observations from these combinations are shown only for the month of January. It is evident that the BiLSTM network provides lesser RMSE of $31.91 \mathrm{~W} / \mathrm{m}^{2}$ and MAPE of $3.43 \%$ with the combinations A7, D7 and sum (D1-D6). In this combination, the detail components D1-D6 are added and resultant subseries is considered as a single input feature to the model instead of six subseries (D1 to D6).

Finally, A7, D7, sum (D1-D6) with time lag input series are used as an input features to the BiLSTM network to forecast the solar GHI. It is evident from Tables 2, 3, 4, this model obtained MAPE ranges from 2.39-17.46\%, RMSE ranges from $23.78-106.65 \mathrm{~W} / \mathrm{m}^{2}$ and $\mathrm{R}^{2}$ ranges from 0.73-0.98. Moreover, the average annual RMSE obtained from the same is $50.36\left(\mathrm{~W} / \mathrm{m}^{2}\right)$, MAPE is $7.28 \%$ and $\mathrm{R}^{2}$ is 0.92 . From the results, it can be seen that this model improves the forecasting accuracy in comparison to naïve, LSTM, GRU, BiLSTM, and WT-BiLSTM (T).

\section{\# Scenario 4: Forecast using proposed WT BiLSTM Model (WT-BiLSTM (CF))}

The proposed model is implemented in this scenario with an aim to achieve credible improvements in the forecasting accuracy. Unlike, all above experiments, this experiment allocated the separate BiLSTM network to each of the resultant decomposed series i.e. A7, D7 and Sum (D1 to D6) to forecast the future subseries's values. The fifteen-time lags of every decomposed component series are considered as the input features to their respective networks. The final prediction is obtained by the wavelet reconstruction process where outputs of each BiLSTM networks are further added to each other. From Tables 2 and 3 , the MAPE obtained by the proposed model ranges from $2.02-14.66 \%$ whereas RMSE is from $21.24-96.38 \mathrm{~W} / \mathrm{m}^{2}$. Table 4 shows the value obtained for $\mathrm{R}^{2}$ which is ranges from 0.78-0.98. Moreover, this model achieved the lowest annual average RMSE $\left(45.61\left(\mathrm{~W} / \mathrm{m}^{2}\right)\right)$, MAPE $(6.48 \%)$ and $\mathrm{R}^{2}(0.94)$.

\section{Discussion}

The research has been conducted to forecast the 24-h ahead solar GHI for the location of Ahmadabad, Gujarat, India in which various experiments are carried out to obtain the precise forecast model with improved forecasts. In order to show the superiority of proposed model, the forecasting performance has been compared with naïve predictor, unidirectional LSTM, unidirectional GRU, BiLSTM and waveletbased BiLSTM models.

From the prospective of overall results,

Table 5 Analysis of WT components combinations as an input features

\begin{tabular}{llllll}
\hline Combination & RMSE $\left(\mathrm{W} / \mathrm{m}^{2}\right)$ & MAPE $(\%)$ & Combination & RMSE $\left(\mathrm{W} / \mathrm{m}^{2}\right)$ & MAPE $(\%)$ \\
\hline A7, D7, D6, D5, D4, D3,D2, D1 & 32.52 & 3.66 & A7, D7, D6, D5, D4, D3, Sum(D1-D2) & 32.49 & 3.82 \\
A7, D7, D6, D5, D4, D3, D2 & 34.75 & 3.86 & A7, D7, D6, D5, D4, Sum (D1-D3) & 34.37 & 3.72 \\
A7, D7, D6, D5, D4, D3 & 34.90 & 3.80 & A7, D7, D6, D5, Sum (D1-D4) & 33.92 & 3.80 \\
A7, D7, D6, D5, D4 & 34.95 & 3.99 & A7, D7, D6, Sum (D1-D5) & 32.35 & 3.69 \\
A7, D7, D6, D5 & 33.02 & 3.82 & A7, D7, Sum (D1-D6) & 31.91 & 3.07 \\
A7, D7, D6 & 35.22 & 3.99 & A7, Sum (D1-D7) & 3.54 \\
A7, D7 & 32.72 & 3.75 & & & \\
\hline
\end{tabular}


a) Naïve predictor provides the largest annual average RMSE of $86.33 \mathrm{~W} / \mathrm{m}^{2}$ and MAPE of $11.74 \%$ due to poorest RMSE in months of February, June-September. The $\mathrm{R}^{2}$ is also lesser in case of naïve predictor which represents the worst forecasting accuracy among all models.

b) As far as, the standalone models are concerned, BiLSTM is the best performer among the naïve, LSTM \& GRU. The annual average RMSE, MAPE $\& \mathrm{R}^{2}$ obtained by BiLSTM is $56.03 \mathrm{~W} / \mathrm{m}^{2}, 8.02 \%$ \& $0.91 \%$ respectively. Whereas, among naïve, GRU and LSTM; GRU performed well than the LSTM \& benchmark model with annual average RMSE, MAPE \& $\mathrm{R}^{2}$ of $77.03 \mathrm{~W} / \mathrm{m}^{2}$, $12.84 \%$ and 0.82 respectively.

c) The traditional WT based BiLSTM model (WT-BiLSTM (T)) performed better than the standalone deep learning based \& naïve models. It obtained $51.77 \mathrm{~W} /$ $\mathrm{m}^{2}$ of annual average RMSE, $7.49 \%$ annual average MAPE and $0.91 \mathrm{R}^{2}$. However, the WT-BiLSTM (mod) model achieved the next level lowest annual average RMSE $\left(50.36 \mathrm{~W} / \mathrm{m}^{2}\right)$ and annual average MAPE $(7.28 \%)$. This model obtained the $\mathrm{R}^{2}$ equals to the 0.92 .

d) Finally, the proposed model performed the best with lowest annual average RMSE $\left(45.61 \mathrm{~W} / \mathrm{m}^{2}\right)$, annual average MAPE (6.48\%). The credible improvement can also be seen in the coefficient of determination $\mathrm{R}^{2}$ (0.94). From the Fig. 9, it is evident that the maximum percentage improvement is provided by the proposed model in RMSE \& MAPE is $57 \%$ \& $58.92 \%$ respectively concerning to standalone LSTM model. However, this improvement is $56.06 \%$ \& $59.19 \%$ respectively against standalone GRU and $31.35 \%$ \& $28.14 \%$ respectively against standalone BiLSTM model. Even, the proposed model also shows a great percentage improvement in RMSE against the naïve predictor ranges from $26.04 \%-$ $58.89 \%$. It provides the great improvements of above $40 \%$ in the eight months i.e. Jan, Feb, March, July, Aug, Sep, Nov and December.
From the month wise prospective,

a) From the literature, no doubt naïve predictor provides the good accuracy for the shorter time horizon with lesser variations in the dataset. However, it is not suitable for the larger time horizon as well as the dataset having larger variations. From the results, it is obvious that the naïve predictor performed better than the LSTM in the month of April, May \& November while better than GRU in month of April and November with a minor difference in RMSE. The MAPE obtained by naïve predictor in month of January, March April, May, July, September, October, November and December are better than LSTM \& GRU. These lowest results are however, uncertain but due to the variations in the datasets.

b) On the other hand, in comparison to the traditional standalone LSTM, GRU \& BiLSTM models, the performance of standalone BiLSTM model is better than the LSTM \& GRU for all months. The standalone BiLSTM model obtained minimum RMSE of $30.94 \mathrm{~W} / \mathrm{m}^{2}$ for the month of December \& MAPE of $2.51 \%$ for the month of April. Moreover, the $\mathrm{R}^{2}$ for BiLSTM model is also better for each month. Even, the naïve predictor, LSTM \& GRU model obtained the worst $\mathrm{R}^{2}$ for the month of July, August \& September i.e. $0.46,0.33,0.56$ for naïve, $0.43,0.40,0.58$ for LSTM and $0.43,0.41,0.47$ for GRU. The results demonstrated that the $\mathrm{R}^{2}$ for these three months are drastically improved by BiLSTM model i.e. $0.81,0.69$, and 0.86. So, the standalone BiLSTM model improves the forecasting accuracy as compared to standalone unidirectional LSTM, GRU and naïve predictor due to its learning capabilities in both directions.

c) By contrast, the wavelet-based models produced high accuracy with lesser error than the standalone models. The traditional wavelet based BiLSTM model performed better than all standalone models including naïve predictor for all months due to extraction of statistical features of input series. It obtained lowest RMSE \& MAPE of $29.86\left(\mathrm{~W} / \mathrm{m}^{2}\right)$ and $2.39 \%$ respectively in month of
Fig. 9 Percentage improvement by proposed model over different models

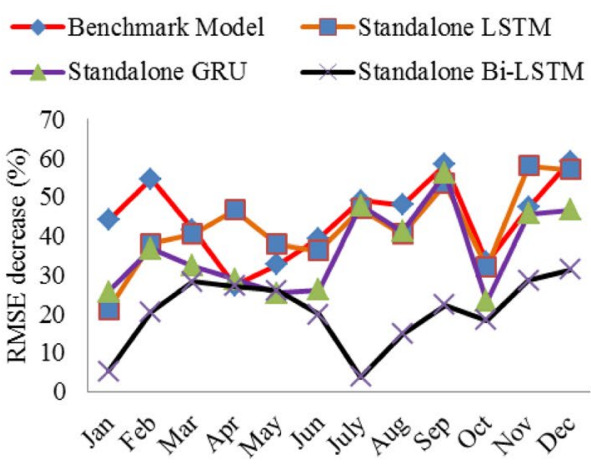

(a)

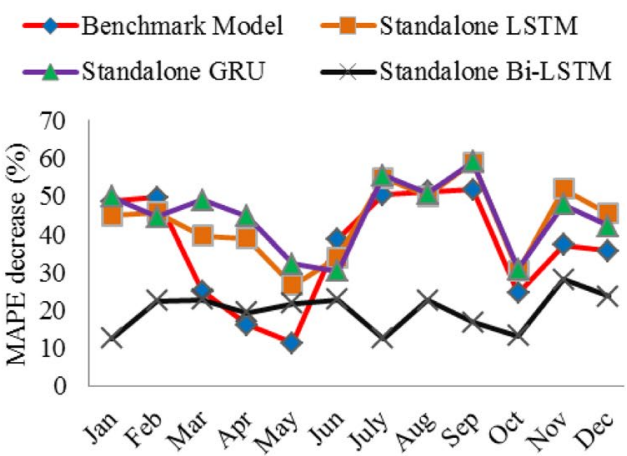

(b) 
April. The maximum RMSE \& MAPE obtained by WT$\operatorname{BiLSTM}(\mathrm{T})$ is $107.17\left(\mathrm{~W} / \mathrm{m}^{2}\right)$ and $17.11 \%$ in month of August. The maximum $\mathrm{R}^{2}(0.98)$ is achieved by the WT-BiLSTM (T) for the month of December.

d) It is also evident from the results that the modified approach of WT with the summation of decomposed components D1-D6 improved the forecasting accuracy than a traditional WT. The lowest RMSE is $23.78 \mathrm{~W} /$ $\mathrm{m}^{2}$ for the month of December \& maximum RMSE is $106.65 \mathrm{~W} / \mathrm{m}^{2}$ for the month of august for the modified WT i.e. WT-BiLSTM (mod). Whereas, it is $25.53 \mathrm{~W} /$ $\mathrm{m}^{2} \& 107.17 \mathrm{~W} / \mathrm{m}^{2}$ for a traditional WT respectively for the same months. However, the $\mathrm{R}^{2}$ value for the month December for WT-BiLSTM (mod) is same as WT$\operatorname{BiLSTM}(\mathrm{T})$ i.e. 0.98 but the improvement was obtained in month of august i.e. 0.73 for WT-BiLSTM(mod) and 0.72 for WT-BiLSTM(T).

e) Finally, the proposed model where each component of WT was separately forecasted using BiLSTM, performed better than all other models. The proposed model obtained lowest RMSE $\left(21.24 \mathrm{~W} / \mathrm{m}^{2}\right)$ for month of December and maximum RMSE $\left(96.38 \mathrm{~W} / \mathrm{m}^{2}\right)$ for the month of August. Whereas, the MAPE obtained by the proposed model is also lesser than others i.e. minimum MAPE of $2.02 \%$ and maximum MAPE of $14.66 \%$. Moreover, the $\mathrm{R}^{2}$ value is also improved in month of august i.e. 0.78 which was initially 0.33 for naïve predictor. The maximum $\mathrm{R}^{2}(0.98)$ is achieved by the proposed Model for the months of March, April, November and December.

For a deep look into the results, the Fig. 10 provides the graphical analysis of real and forecasted GHI for three consecutive days (2nd to 4th day) of two months (March $\&$ August). These two months are to visualize the results of month with lesser RMSE (March) and larger RMSE (August). Nevertheless, for a clear presentation, only real GHI and forecasted GHI curves of proposed model is presented for selected months. It is clear from the Fig. 10; the large variations in the real GHI curve provided the larger error in the results. For instance, the month of March has a smooth curve due to clear sky conditions and easily traceable by the model. Unlike this month, the real GHI is frequently varying in the month of August due to presence of cloudy or rainy days, and is hard to trace by the model results in maximum errors. From these curve traces, it can be concluded that higher is the variations in real GHI, lower is the similarity in real and forecasted GHI. Similarly, lower are the variations in the real GHI; higher is the degree of similarity between real and forecasted GHI. But, the proposed model also follows uncertainties associated with real GHI with in tolerance band of error. Therefore, these observations yield that the proposed model is a suitable forecasting model not only for stable type of seasons but also for unstable seasons.

For further explanation of forecasting performance, Table 6 shows the FS\% results of proposed model and WT-BiLSTM (mod) for annual RMSE \& MAPE with reference to benchmark model.

Lastly, to validate the performance of the proposed model against previously reported models is presented in Table 7. The superiority of the proposed model is shown in terms of RMSE, MAPE and FS.

According to the Table 7, the proposed model appears to be the best and excellent model to forecast solar GHI over latest developed models. The model performed shows its superiority in terms of lower RMSE \& MAPE and higher forecasting skills over LSTM, GRU, CNN-LSTM, DFT-PCA-Elman and standalone BiLSTM. Therefore, the overall results suggested that the proposed model can be a good choice to forecast solar GHI for practical solar power systems also.

Table 6 Annual \%FS of proposed model

\begin{tabular}{lll}
\hline Model & $\mathrm{FS}_{\text {RMSE }}$ & $\mathrm{FS}_{\text {MAPE }}$ \\
\hline WT-BiLSTM(mod) & $42 \%$ & $38 \%$ \\
Proposed Model & $47 \%$ & $45 \%$ \\
\hline
\end{tabular}

Fig. 10 a, b Performance of proposed model for month March \& August respectively

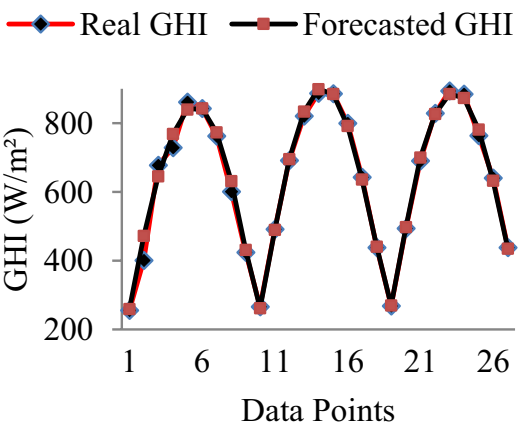

(a) March

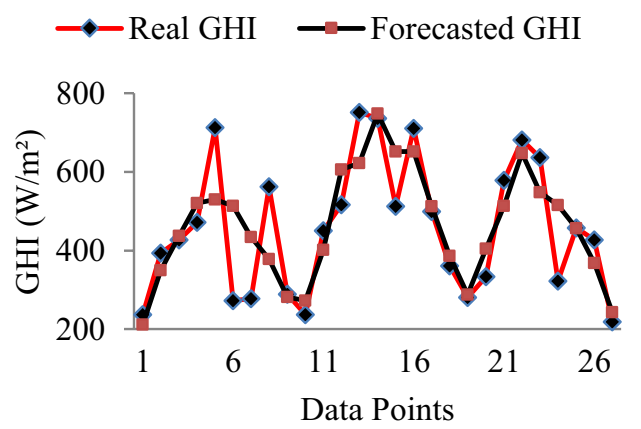

(b) August 
Table 7 Comparison of proposed Model to the previously developed models

\begin{tabular}{|c|c|c|c|c|c|c|c|}
\hline Ref. & YOP & Location & Time horizon & Model & $\operatorname{RMSE}\left(\mathrm{W} / \mathrm{m}^{2}\right)$ & MAPE (\%) & FS $\%$ \\
\hline (Qing and Niu 2018) & 2018 & Santiago, Cape Verde & $1-\mathrm{h}$ & LSTM & 126.31 & - & - \\
\hline (Lan et al. 2019) & 2019 & Qingdao, China & 24-h & $\mathrm{DFT}+\mathrm{PCA}+$ Elman/BPNN & 127.3 & & 6.3 \\
\hline (Gao et al. 2019) & 2019 & Denver, USA & 24-h & GRU & 122.45 & - & 42.01 \\
\hline (Zang et al. 2020b) & 2020 & Texas, USA & 1-h & CNN-LSTM & 69.26 & - & - \\
\hline (Kumari and Toshniwal 2021) & 2020 & New Delhi, India & 1-h & XGBF-DNN & 51.35 & - & 40.2 \\
\hline (Li et al. 2021) & 2021 & us & 1-h & BiLSTM & 98.44 & - & - \\
\hline This Work & & Ahmadabad, India & 24-h & $W T-L S T M(\mathrm{CF})$ & 45.61 & 6.48 & 47 \\
\hline
\end{tabular}

\section{Conclusion}

In the renewable energy markets, solar energy has its own space \& significance. The stability and efficiency of solar forecasting are therefore crucial for the regular operation of interconnected grid systems. Most of the time, the standalone models are unable to capture the fluctuating inherent solar time series characteristics. So, an ensemble model to forecast the 24-h ahead hourly solar GHI is proposed using the WT and BiLSTM network. The performance of the proposed model is rigorously evaluated by the GHI forecast on monthly basis for a year 2014 using moving window. The WT is used to extract the statistical features of the input time series using low and high frequency time series (IMF) while BiLSTM network is employed to the forecast. Meanwhile, the forecasting performance of the proposed model is compared with the naïve predictor, standalone LSTM, GRU, BiLSTM and two different WT based BiLSTM model. The results show that the proposed model outperforms the other models with minimum annual average RMSE $\left(45.61 \mathrm{~W} / \mathrm{m}^{2}\right)$ and MAPE $(6.48 \%)$. The forecast skill and the $\mathrm{R}^{2}$ for the proposed model are $47 \%$ and 0.94 respectively. Finally, the maximum percentile improvement in RMSE by proposed model is $58.38 \%$, $58.11 \%, 56.06 \%$ \& $31.35 \%$ over benchmark model, standalone LSTM, GRU and BiLSTM respectively; whereas, maximum percentile improvement in MAPE is $51.76 \%$, $58.92 \%, 59.19 \%$ \& $28.14 \%$ over benchmark model, standalone LSTM, GRU and BiLSTM respectively. Therefore, proposed model is proved to be the best forecasting model for 24-h ahead solar forecasts with consideration of all seasonal as well as monthly aspects. However, the study observed some challenges while designing the models such as: selection of precise hyperparameters and simulation time. So, in future by considering these challenges, more accurate and efficient results can be obtained within quick simulation time.

Data availability The data that support the findings of this study are available from the corresponding author upon reasonable request.
Code availability The codes of this study are available from the corresponding author upon reasonable request.

\section{Declarations}

Not Applicable.

Conflict of interest On behalf of all authors, the corresponding author states that there is no conflict of interest.

\section{References}

Aguiar LM, Pereira B, Lauret P, Díaz F, David M (2016) Combining solar irradiance measurements, satellite-derived data and a numerical weather prediction model to improve intra-day solar forecasting. Renew Energy 97:599-610. https://doi.org/10.1016/j. renene.2016.06.018

Alonso-Montesinos J, Batlles FJ (2015) Solar radiation forecasting in the short- and medium-term under all sky conditions. Energy 83:387-393. https://doi.org/10.1016/j.energy.2015.02.036

AlSkaif T, Dev S, Visser L, Hossari M, van Sark W (2020) A systematic analysis of meteorological variables for PV output power estimation. Renew Energy 153:12-22. https://doi.org/10.1016/j. renene.2020.01.150

Bao Y, Huang Z, Li L, Wang Y, Liu Y (2020) A BiLSTM-CNN model for predicting users' next locations based on geotagged social media. Int J Geogr Inf Sci 35(4):639-660. https://doi.org/10.1080/ 13658816.2020.1808896

Bedi J, Toshniwal D (2019) Deep learning framework to forecast electricity demand. Appl Energy 238:1312-1326. https://doi.org/10. 1016/j.apenergy.2019.01.113

Benali L, Notton G, Fouilloy A, Voyant C, Dizene R (2019) Solar radiation forecasting using artificial neural network and random forest methods: application to normal beam, horizontal diffuse and global components. Renew Energy 132:871-884. https://doi.org/ 10.1016/j.renene.2018.08.044

Bigdeli N, Salehi Borujeni M, Afshar K (2017) Time series analysis and short-term forecasting of solar irradiation, a new hybrid approach. Swarm Evol Comput 34:75-88. https://doi.org/10. 1016/j.swevo.2016.12.004

Chen B, Lin P, Lai Y, Cheng S, Chen Z, Wu L (2020) Very-short-term power prediction for $\mathrm{PV}$ power plants using a simple and effective RCC-LSTM model based on short term multivariate historical datasets. Electronics 9(2):289. https://doi.org/10.3390/electronic s9020289

Cheng H, Ding X, Zhou W, Ding R (2019) A hybrid electricity price forecasting model with Bayesian optimization for German energy exchange. Int J Electr Power Energy Syst 110:653-666. https:// doi.org/10.1016/j.ijepes.2019.03.056

Cornejo-Bueno L, Casanova-Mateo C, Sanz-Justo J, Salcedo-Sanz S (2019) Machine learning regressors for solar radiation estimation 
from satellite data. Sol Energy 183:768-775. https://doi.org/10. 1016/j.solener.2019.03.079

Ding M, Zhou H, Xie H, Wu M, Nakanishi Y, Yokoyama R (2019) A gated recurrent unit neural networks based wind speed error correction model for short-term wind power forecasting. Neurocomputing 365:54-61. https://doi.org/10.1016/j.neucom.2019.07.058

Dong N, Chang JF, Wu AG, Gao ZK (2020) A novel convolutional neural network framework based solar irradiance prediction method. Int J Electr Power Energy Syst 114:105411. https://doi.org/10. 1016/j.ijepes.2019.105411

Doorga JRS, Rughooputh SDDV, Boojhawon R (2019) Modelling the global solar radiation climate of Mauritius using regression techniques. Renew Energy 131:861-878. https://doi.org/10.1016/j. renene.2018.07.107

Dumitru C-D, Gligor A, Enachescu C (2016) Solar photovoltaic energy production forecast using neural networks. Procedia Technol 22:808-815. https://doi.org/10.1016/j.protcy.2016.01.053

El-Hendawi M, Wang Z (2020) An ensemble method of full wavelet packet transform and neural network for short term electrical load forecasting. Electr Power Syst Res 182:106265. https://doi.org/10. 1016/j.epsr.2020.106265

Feng C, Zhang J (2020) SolarNet: a sky image-based deep convolutional neural network for intra-hour solar forecasting. Sol Energy 204:71-78. https://doi.org/10.1016/j.solener.2020. 03.083

Fischer T, Krauss C (2018) Deep learning with long short-term memory networks for financial market predictions. Eur J Oper Res 270(2):654-669. https://doi.org/10.1016/j.ejor.2017.11.054

Gao B, Huang X, Shi J, Tai Y, Xiao R (2019) Predicting day-ahead solar irradiance through gated recurrent unit using weather forecasting data. J Renew Sustain Energy 11(4):043705. https://doi. org/10.1063/1.5110223

Gao B, Huang X, Shi J, Tai Y, Zhang J (2020) Hourly forecasting of solar irradiance based on CEEMDAN and multi-strategy CNNLSTM neural networks. Renew Energy 162:1665-1683. https:// doi.org/10.1016/j.renene.2020.09.141

Hales D, Renewables (2018) Global. Status report 2018

Heydari A, Astiaso Garcia D, Keynia F, Bisegna F, De Santoli L (2019) A novel composite neural network based method for wind and solar power forecasting in microgrids. Appl Energy 251. https:// doi.org/10.1016/j.apenergy.2019.113353

Hochreiter S, Schmidhuber J (1997) Long short-term memory. Neural Comput 9(8):1735-1780. https://doi.org/10.1162/neco.1997.9.8. 1735

Hu YL, Chen L (Oct. 2018) A nonlinear hybrid wind speed forecasting model using LSTM network, hysteretic ELM and differential evolution algorithm. Energy Convers Manag 173:123-142. https:// doi.org/10.1016/j.enconman.2018.07.070

Huang C, Wang L, Lai LL (2019) Data-driven short-term solar irradiance forecasting based on information of neighboring sites. IEEE Trans Ind Electron 66(12):9918-9927. https://doi.org/10.1109/ TIE.2018.2856199

Jacobson MZ, Delucchi MA, Bauer ZAF et al (2017) 100\% clean and renewable wind, water, and sunlight all-sector energy roadmaps for 139 countries of the world. Joule 1(1):108-121. https://doi. org/10.1016/j.joule.2017.07.005

Jahani B, Mohammadi B (2019) A comparison between the application of empirical and ANN methods for estimation of daily global solar radiation in Iran. Theor Appl Climatol 137(1-2):1257-1269. https://doi.org/10.1007/s00704-018-2666-3

Kim J, Moon N (2019) BiLSTM model based on multivariate time series data in multiple field for forecasting trading area. J Ambient Intell Humaniz Comput:1-10. https://doi.org/10.1007/ s12652-019-01398-9
Kulshrestha A, Krishnaswamy V, Sharma M (2020) Bayesian BILSTM approach for tourism demand forecasting. Ann Tour Res 83:102925. https://doi.org/10.1016/j.annals.2020.102925

Kumar KR, Kalavathi MS (2018) Artificial intelligence based forecast models for predicting solar power generation. Materials Today: Proceedings 5(1):796-802. https://doi.org/10.1016/j.matpr.2017. 11.149

Kumar D, Mathur HD, Bhanot S, Bansal RC (2020) Forecasting of solar and wind power using LSTM RNN for load frequency control in isolated microgrid. Int J Model Simul. https://doi.org/10. 1080/02286203.2020.1767840

Kumari P, Toshniwal D (2021) Extreme gradient boosting and deep neural network based ensemble learning approach to forecast hourly solar irradiance. J Clean Prod 279:123285. https://doi.org/ 10.1016/j.jclepro.2020.123285

Kumler A, Xie Y, Zhang Y (2019) A physics-based smart persistence model for intra-hour forecasting of solar radiation (PSPI) using GHI measurements and a cloud retrieval technique. Sol Energy 177:494-500. https://doi.org/10.1016/j.solener.2018.11.046

Labouret A, Villoz M (2010) Solar photovoltaic energy. Sol Photovolt Energy:1-373. https://doi.org/10.1049/pbrn009e

Lai C, Li J, Chen B, Huang Y, Wei S (2019) Review of photovoltaic power output prediction technology. Diangong Jishu Xuebao/ Transactions of China Electrotechnical Society 34(6) Chinese Machine Press:1201-1217. https://doi.org/10.19595/j.cnki.10006753.tces. 180326

Lai JP, Chang YM, Chen CH, Pai PF (2020) A survey of machine learning models in renewable energy predictions. Appl Sci 10(17). https://doi.org/10.3390/app10175975

Lan H, Zhang C, Hong YY, He Y, Wen S (2019) Day-ahead spatiotemporal solar irradiation forecasting using frequency-based hybrid principal component analysis and neural network. Appl Energy 247:389-402. https://doi.org/10.1016/j.apenergy.2019.04.056

Lauret P, Voyant C, Soubdhan T, David M, Poggi P (2015) A benchmarking of machine learning techniques for solar radiation forecasting in an insular context. Sol Energy 112:446-457. https://doi. org/10.1016/j.solener.2014.12.014

Li K, Zhang T (2019) A novel grey forecasting model and its application in forecasting the energy consumption in Shanghai. Energy Syst:1-16. https://doi.org/10.1007/s12667-019-00344-0

Li C, Zhang Y, Zhao G, Ren Y (2021) Hourly solar irradiance prediction using deep BiLSTM network. Earth Sci Informatics 14:299309. https://doi.org/10.1007/s12145-020-00511-3

Liu D, Sun K (2019) Random forest solar power forecast based on classification optimization. Energy 187. https://doi.org/10.1016/j. energy.2019.115940

Mishra M, Byomakesha Dash P, Nayak J, Naik B, Kumar Swain S (2020) Deep learning and wavelet transform integrated approach for short-term solar PV power prediction. Meas J Int Meas Confed 166:108250. https://doi.org/10.1016/j.measurement.2020.108250

Mohammadi B, Aghashariatmadari Z (2020) Estimation of solar radiation using neighboring stations through hybrid support vector regression boosted by Krill Herd algorithm. Arab J Geosci 13(10):1-16. https://doi.org/10.1007/s12517-020-05355-1

Perez R, Kivalov S, Schlemmer J, Hemker K, Hoff TE (2012) Shortterm irradiance variability: Preliminary estimation of station pair correlation as a function of distance. Solar Energy 86(8) Pergamon:2170-2176. https://doi.org/10.1016/j.solener.2012.02.027

Qing X, Niu Y (2018) Hourly day-ahead solar irradiance prediction using weather forecasts by LSTM. Energy 148:461-468. https:// doi.org/10.1016/j.energy.2018.01.177

Rai A, Shrivastava A, Jana KC (2021) A CNN-BiLSTM based deep learning model for mid-term solar radiation prediction. Int Trans Electr Energy Syst 11(18):8613. https://doi.org/10.3390/app11 188613 
Reza Parsaei M, Mollashahi H, Darvishan A, Mir M, Simoes R (2020) A new prediction model of solar radiation based on the neurofuzzy model. Int J Ambient Energy 41(2):189-197. https://doi. org/10.1080/01430750.2018.1456964

Richardson DS, Cloke HL, Pappenberger F (2020) Evaluation of the consistency of ECMWF ensemble forecasts. Geophys Res Lett 47(11). https://doi.org/10.1029/2020GL087934

Ruhang X (2016) The restriction research for urban area building integrated grid-connected PV power generation potential. Energy 113:124-143. https://doi.org/10.1016/j.energy.2016.07.035

Saroha S, Aggarwal SK (2018) Wind power forecasting using wavelet transforms and neural networks with tapped delay. CSEE J. Power Energy Syst 4(2):197-209. https://doi.org/10.17775/cseej pes.2016.00970

Shadab A, Ahmad S, Said S (2020) Spatial forecasting of solar radiation using ARIMA model. Remote Sens Appl Soc Environ 20:100427. https://doi.org/10.1016/j.rsase.2020.100427

Shamshirband S, Mohammadi K, Khorasanizadeh H et al (2016) Estimating the diffuse solar radiation using a coupled support vector machine-wavelet transform model. Renew Sustain Energy Rev 56. Elsevier Ltd:428-435. https://doi.org/10.1016/j.rser.2015.11.055

Sheng H, Xiao J, Cheng Y, Ni Q, Wang S (2018) Short-term solar power forecasting based on weighted Gaussian process regression. IEEE Trans Ind Electron 65(1):300-308. https://doi.org/10.1109/ TIE.2017.2714127

Singh S, Hussain S, Bazaz MA (2018) Short term load forecasting using artificial neural network. In: 2017 4th International Conference on Image Information Processing, ICIIP 2017, Mar. 2018, vol. 2018-January, pp. 159-163. https://doi.org/10.1109/ICIIP. 2017.8313703

Sobri S, Koohi-Kamali S, Rahim NA (2018) Solar photovoltaic generation forecasting methods: A review. Energy Conversion and Management 156. Elsevier Ltd:459-497. https://doi.org/10.1016/j. enconman.2017.11.019

Wang F, Yu Y, Zhang Z, Li J, Zhen Z, Li K (2018) Wavelet decomposition and convolutional LSTM networks based improved deep learning model for solar irradiance forecasting. Appl Sci 8(8):1286. https://doi.org/10.3390/app8081286

Wang H, Lei Z, Zhang X, Zhou B, Peng J (2019) A review of deep learning for renewable energy forecasting. Energy Conversion and Management 198. Elsevier Ltd:111799. https://doi.org/10.1016/j. enconman.2019.111799

Wu K, Wu J, Feng L et al (2020) An attention-based CNN-LSTM-BiLSTM model for short-term electric load forecasting in integrated

\section{Authors and Affiliations}

\section{Pardeep Singla $^{1}$ (D) $\cdot$ Manoj Duhan ${ }^{1} \cdot$ Sumit Saroha ${ }^{2}$}

1 Deenbandhu Chhotu Ram University of Science \& Technology, Sonepat, India energy system. Int Trans Electr Energy Syst. https://doi.org/10. 1002/2050-7038.12637

Yang H, Kleissl J (2016) Preprocessing WRF initial conditions for coastal stratocumulus forecasting. Sol Energy 133:180-193. https://doi.org/10.1016/j.solener.2016.04.003

Yildirim Ö (2018) A novel wavelet sequences based on deep bidirectional LSTM network model for ECG signal classification. Comput Biol Med 96:189-202. https://doi.org/10.1016/j.compb iomed.2018.03.016

Yousif C, Quecedo GO, Santos JB (2013) Comparison of solar radiation in Marsaxlokk, Malta and Valladolid, Spain. Renew Energy 49:203-206. https://doi.org/10.1016/j.renene.2012.01.031

Yu Y, Cao J, Zhu J (2019) An LSTM short-term solar irradiance forecasting under complicated weather conditions. IEEE Access 7:145651-145666. https://doi.org/10.1109/ACCESS.2019.29460 57

Zang H, Cheng L, Ding T, Cheung KW, Wei Z, Sun G (2020a) Dayahead photovoltaic power forecasting approach based on deep convolutional neural networks and meta learning. Int J Electr Power Energy Syst 118:105790. https://doi.org/10.1016/j.ijepes. 2019.105790

Zang H, Liu L, Sun L, Cheng L, Wei Z, Sun G (2020b) Short-term global horizontal irradiance forecasting based on a hybrid CNNLSTM model with spatiotemporal correlations. Renew Energy 160:26-41. https://doi.org/10.1016/j.renene.2020.05.150

Zeng J, Qiao W (2013) Short-term solar power prediction using a support vector machine. Renew Energy 52:118-127. https://doi.org/ 10.1016/j.renene.2012.10.009

Zeroual A, Harrou F, Dairi A, Sun Y (2020) Deep learning methods for forecasting COVID-19 time-series data: a comparative study. Chaos, Solitons and Fractals 140:110121. https://doi.org/ 10.1016/j.chaos.2020.110121

Zhang T, Lv C, Ma F, Zhao K, Wang H, O'Hare GMP (2020) A photovoltaic power forecasting model based on dendritic neuron networks with the aid of wavelet transform. Neurocomputing 397:438-446. https://doi.org/10.1016/j.neucom.2019.08.105

Publisher's note Springer Nature remains neutral with regard to jurisdictional claims in published maps and institutional affiliations.

2 Guru Jambheshwar University of Science and Technology, Hisar, India 